\title{
Rapid Mobility of Motile Varicosities and Inclusions Containing $\alpha$-Spectrin, Actin, and Calmodulin in Regenerating Axons In Vitro ${ }^{1}$
}

\author{
E. KOENIG, ${ }^{2}$ S. KINSMAN, E. REPASKY, ${ }^{\star}$ AND L. SULTZ
}

Division of Neurobiology, Department of Physiology, State University of New York, Buffalo, New York 14214 and ${ }^{\star}$ Department of Molecular Immunology, Roswell Park Memorial Institute, Buffalo, New York 14263

\begin{abstract}
Time-lapse video recording was used to investigate the bidirectional movements of motile varicosities and intervening phase-dense (IPD) inclusions associated with axons of goldfish retinal ganglion cells regenerating in vitro. In addition, analyses of fine structure and immunocytochemical distributions of $\alpha$-spectrin (fodrin), actin, and calmodulin in axonal fields were undertaken. Varicosities and IPD inclusions undergo saltations in a random manner at mean rates of 0.218 and $3.33 \mu \mathrm{m} / \mathrm{sec}$, respectively. Experiments involving calcium antagonists or depletion of internal calcium stores resulted in an arrest of all intra-axonal movement, indicating that saltations of the two mobile structures are dependent on intra-axonal calcium. The predominant structure in varicosities is a large aggregation of an anastomosing, tubular, smooth endoplasmic reticulum embedded in an amorphous matrix, suggesting a form of "packaged" cytomembranes undergoing bulk transport. IPD inclusions, presumably carrying membranes, appear to shuttle between varicosities and growth cones during axon elongation, and between growth cones and varicosities during axon retraction. $\alpha$-Spectrin, actin, and calmodulin were shown by immunocytochemistry to be preferentially distributed to varicosities and IPD inclusions. The co-transport of spectrin, actin, and calmodulin with cytomembranes undergoing rapid saltations departs from reported results of radioactive labeling experiments insofar as spectrin, actin, and calmodulin are not normally associated with rapidly transported membrane components in the latter studies. Possible reasons for the discrepancy are discussed. Our results suggest that spectrin and actin may play a role in the packaging and axoplasmic transport of cytomembranes concerned with plasmalemma recycling.
\end{abstract}

The intracellular transport of membranous organelles from their site of formation in the Golgi complex to axon terminals occurs at rapid rates (for review, see Grafstein and Forman, 1980). Generally, axoplasmic transport of membranous organelles has been studied using biochemical markers or radiolabeled constituents to monitor proximodistal or distoproximal displacement. This approach is limited

Received May 25, 1984; Revised August 6, 1984;

Accepted August 10, 1984

${ }^{1}$ We thank Ms. Terry Crumb, Mr. Brian Edmonds, and Mrs. Priscilla Adams for their technical assistance, Mrs. Jean Seiler for typing and Mrs. Julie Lakatos for photography. This research was supported by United States Public Health Service Grant EY 04443 from the National Eye Institute.

${ }^{2}$ To whom correspondence should be addressed. in its usefulness to probe mechanisms that may govern intracellular motility because it is indirect. An approach that permits direct monitoring of movements associated with axoplasmic organelles and of effects of perturbations on the transport process is the use of isolated axons, viewed by phase or interference microscopy in combination with time-lapse cinemicrography or video recording. This approach has been used with increasing frequency in recent years (Smith, 1971; Kirkpatrick et al., 1972; Cooper and Smith, 1974; Breuer et al., 1975; Forman et al., 1977a, b, 1983; Forman and Shain, 1981; Allen et al., 1982a, b; Koles, et al., 1982).

We have employed the technique of time-lapse video recording to investigate the dynamic behavior of motile varicosities and intervening phase-dense inclusions associated with axons of goldfish retinal ganglion cells regenerating in vitro. The varicosities contain a convoluted, tubular endoplasmic reticulum that undergoes bulk transport. In this report we describe the behavioral and structural features of these motile axonal structures and the effects of some selected perturbations that interfere with their movements. In addition, we show by immunocytochemistry that $\alpha$-spectrin, actin, and calmodulin are preferentially associated with these mobile structures.

\section{Materials and Methods}

The goldfish retinal explant system, developed by Landreth and Agranoff (1976), was used in the present study. Details of the explant preparation have been described (Koenig and Adams, 1982). Briefly, 2 weeks after crushing the optic nerve, the retina was isolated from dark-adapted goldfish, chopped into squares $(0.6 \times 0.6 \mathrm{~mm})$ with a Mcllwain tissue chopper, plated out on polylysine-coated no. $2(0.5 \times 3 \mathrm{~cm})$, or no. 1.5 circular $(24.5-\mathrm{mm}$ diameter) coverslips, and cultured in L-15 medium supplemented with $10 \%$ fetal calf serum (Flow Laboratories), 0.02 M HEPES (Sigma), $0.1 \mathrm{~mm} 5$ fluorodeoxyuridine (Sigma), and $0.2 \mathrm{~mm}$ uridine (Sigma). In later experiments the culture medium was supplemented also with methyl cellulose $(0.6 \%)$ because it enhanced axonal outgrowth and increased survival or axonal fields (Johnson and Turner, 1982; see "Results"). Explants were cultured in a humid air atmosphere at $27^{\circ} \mathrm{C}$

Cultured retinal explants, attached to a coverslip, were placed in a microscope viewing chamber. Initially, a simple glass chamber served this purpose, in which the rectangular coverslip containing explants was inverted over a well and the space was filled with the bathing medium. In late experiments, a Dvorak-Stotler chamber (Nicholson Precision Instruments), which requires a circular coverslip, was used. The latter chamber facilitated the exchange of bathing solutions and allowed reasonably good phase images at high magnification. The bathing medium was a modified Cortland physiological saline (Koenig and Adams, 1982), and the composition was as follows: $132 \mathrm{~mm} \mathrm{NaCl}, 5 \mathrm{~mm} \mathrm{KCl}, 3 \mathrm{mM} \mathrm{NaH}_{2} \mathrm{PO}_{4}, 1.6 \mathrm{~mm} \mathrm{MgSO}_{4}, 1.8 \mathrm{~mm}$ $\mathrm{CaCl}_{2}$, and $5.5 \mathrm{~mm}$ glucose. In divalent cation substitution experiments, a phosphate- and sulfate-free modified Cortland solution was used in which chloride was substituted for the divalent anions. Axonal fields were viewed under phase-contrast microscopy with $\times 40$ (numerical aperture (n.a.) $=$ 0.65 ) or $\times 100$ (n.a. $=1.25$, oil immersion) planochromatic objectives (Zeiss) The phase image was displayed on a video monitor (Sanyo), using a video camera (Sanyo) mounted on the microscope, and was recorded with a timelapse video recorder (GYYR TLC2001). Recording was generally in a time- 

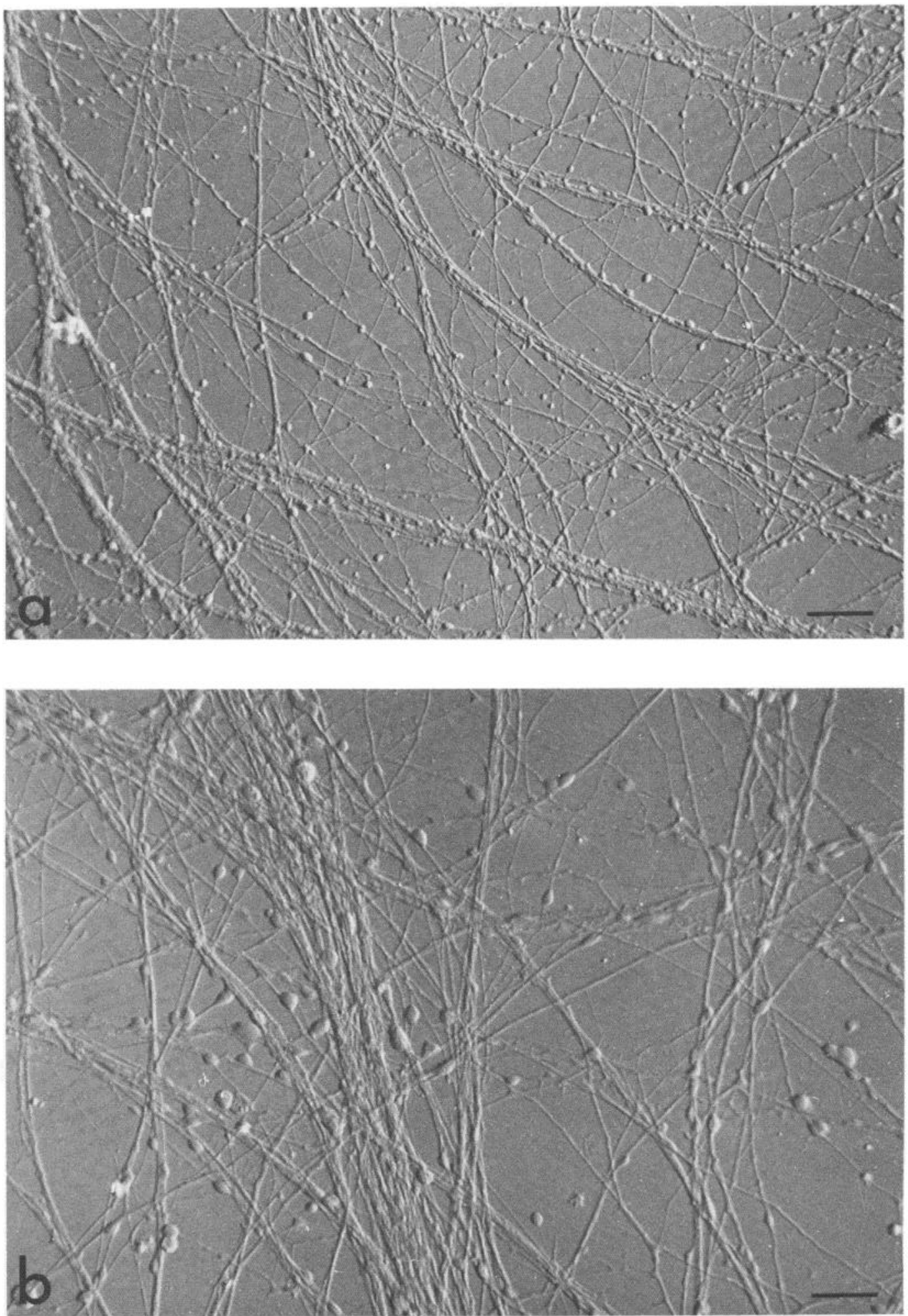

Figure 1. Interference contrast photomicrographs of ganglion cell axons regenerating in vitro from retinal explants. $a$ and $b$ show varicosities distributed randomly in single and fasiculated axons. Calibration bars: $a, 43 \mu \mathrm{m} ; b, 17 \mu \mathrm{m}$.

lapse mode in which time was compressed by a factor of 12 . Observations were conducted at temperatures ranging from 22 to $24^{\circ} \mathrm{C}$.

Axonal fields were prepared for electron microscopy as follows. Explants were washed free of serum with modified Cortland solution, immersed in $2 \%$ glutaraldehyde in $0.1 \mathrm{M}$ sodium cacodylate buffer $(\mathrm{pH} 7.4)$ for $20 \mathrm{~min}$, and then dehydrated in a series of acetone concentrations. Thin sections were cut on a Sorvall MT2B ultramicrotome after imbedding in Epon (Polysciences) and staining with lead citrate and uranyl acetate. Sections were viewed in a JEOL 100-B electron microscope.
Immunofluorescence. The distribution and localization of $\alpha$-spectrin, actin, and calmodulin were investigated by indirect immunocytochemistry. For the localization of $\alpha$-spectrin, we have used an antiserum prepared against chicken erythrocyte $\alpha$-spectrin; details regarding the preparation and characterization of $\alpha$-spectrin antiserum have been described previously (Repasky et al., 1982). We have recently demonstrated by immunochemical and cytochemical techniques that an $\alpha$-spectrin-like polypeptide is present in goldfish axons that co-migrates with authenic chicken erythrocyte $\alpha$-spectrin 


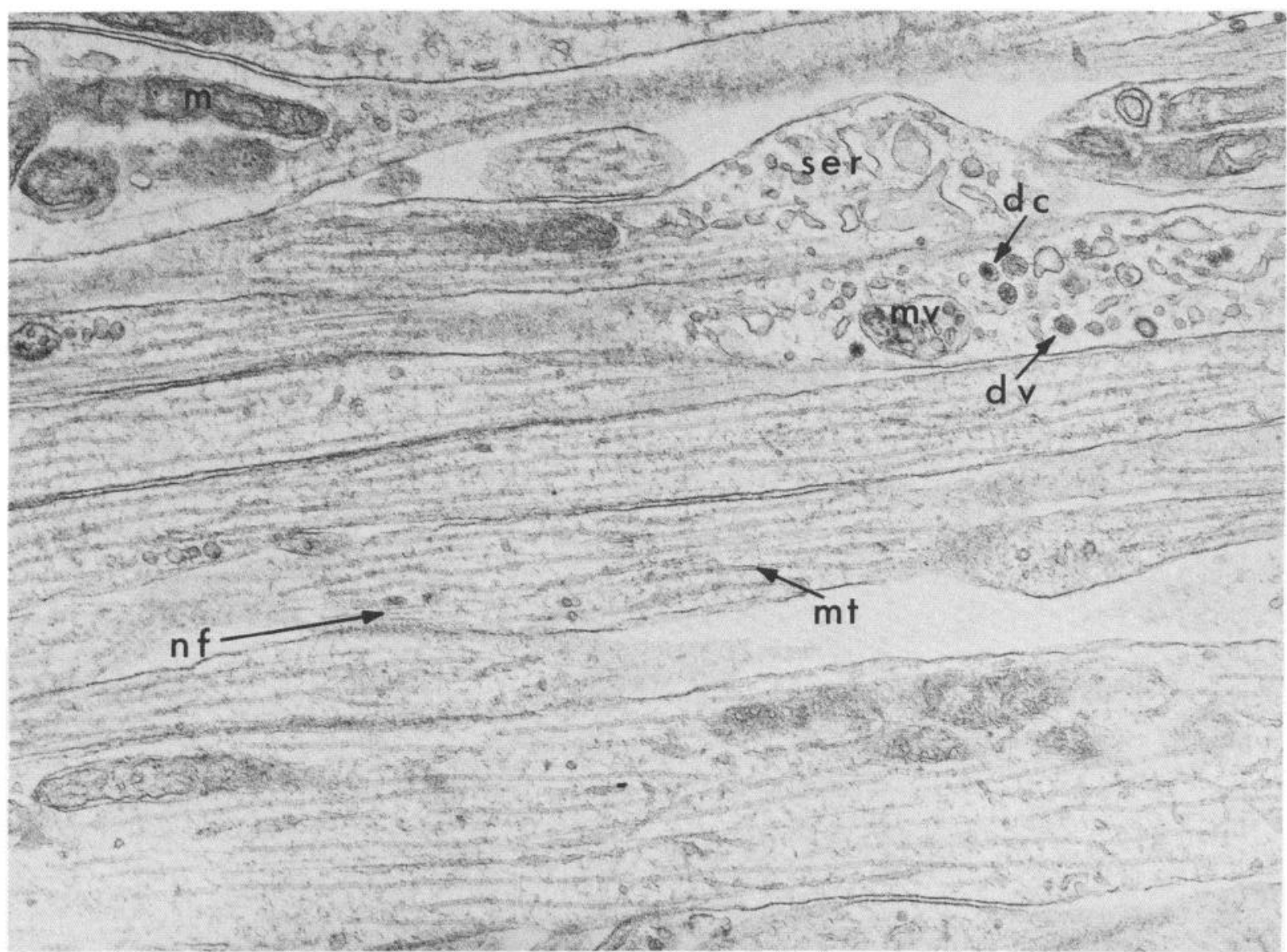

Figure 2. Electron micrograph of axonal segments intervening between varicosities. $d c$, dense-core vesicle; $d v$, dense vesicle; $m$, mitochondrion; $m t$, microtubules; $m v$, multivesicular body; $n f$, neurofilament; ser, smooth endoplasmic reticulum. Magnification $\times 36,600$.

(Koenig and Repasky, 1985). The actin antiserum used in the present study was prepared against chicken gizzard smooth muscle and was kindly provided by Dr. Ute Gröschel-Stewart. In double labeling experiments, rhodamine-conjugated phalloidin (Molecular Probes) was used to localize Factin. Secondary antibodies were goat anti-rabbit or rabbit anti-sheep antibodies. Affinity-purified calmodulin antibodies, raised in sheep against rat testes native calmodulin (Chafouleas, et al., 1979), was purchased from CAABCO, Inc. The same antibody was used recently to demonstrate the association of calmodulin with synaptic vesicles (Hooper and Kelly, 1984).

The preparative procedure for immunofluorescence was as follows. Cultured explants, grown on no. 1 polylysine-coated coverslips, were washed free of culture medium with two rinses of modified Cortland solution in which chloride was substituted for sulfate and phosphate. Explants were fixed by immersion in $1.8 \%$ formaldehyde, $0.1 \mathrm{M}$ sodium cacodylate, and $0.05 \mathrm{M}$ zinc acetate $(\mathrm{pH} 7.4)(10 \mathrm{~min})$. At this juncture axonal fields were severed from the explants and explants were discarded. Axonal fields were extracted with $0.5 \%$ Triton X-100 in $0.1 \mathrm{M}$ zinc acetate, $0.067 \mathrm{M} \mathrm{NaCl}, 2.5 \mathrm{~mm} \mathrm{KCl}, 0.01 \mathrm{M}$ HEPES (pH 6.5). Zinc acetate during the extraction with Triton $X-100$ was helpful in preserving the structural form of varicosities. After washing twice in TM buffer (10 mm Tris- $\mathrm{HCl}, 130 \mathrm{~mm} \mathrm{NaCl}, 5 \mathrm{~mm} \mathrm{KCl}, 5 \mathrm{~mm} \mathrm{NaN}, 5 \mathrm{~mm}$ $\mathrm{MgCl}_{2}, \mathrm{pH} 7.5$ ), the fields were incubated for $1 \mathrm{hr}$ at room temperature with the primary rabbit or sheep antibodies, diluted 1:30 with TM buffer. The fields were washed in TM buffer for $30 \mathrm{~min}$, and then were further incubated with either fluorescein isothiocyanate-conjugated goat anti-rabbit IgG (Sigma) or rhodamine-conjugated rabbit anti-sheep lgG (Cappel), diluted 1:150 with TM buffer. After washing for a minimum of $30 \mathrm{~min}$, the coverslips were either mounted in Elvanol (Rodriguez and Deinhart, 1960) at this point or, in double labeling experiments, incubated for $20 \mathrm{~min}$ with rhodamine-conjugated phalloidin, diluted 1:100 with TM buffer. After two brief washes in TM buffer, the coverslips were mounted and viewed in a Zeiss fluorescence microscope equipped with an epifluorescence attachment.

\section{Results}

Morphology of regenerating axons. The explants were cultured in the presence of 5-fluorodeoxyuridine, which yielded growing axons that remained free of non-neural cells (Heacock and Agranoff, 1977; Koenig and Adams, 1982). The outgrowing axons were frequently associated with meandering fasicles, but many were isolated as single axons. A characteristic morphological feature of these naked axons is the presence of local swellings or varicosities that are distributed with moderate frequency (Fig. 1). Intervening axonal segments between varicosities ordinarily are very thin in proximal axons but are thicker in distal intervening segments contiguous to growth cones (see below).

At the electron microscopic level, the principal structural elements in thin axonal segments intervening between varicosities are microtubules. Other organelles include mitochondria, and less frequently encountered organelles are multivesicular bodies, dense vesicles, dense-core vesicles and smooth tubulovesicular profiles (Fig. 2). There is a paucity of neurofilaments which may have some significance for the manner in which cytomembranes are "packaged" for bulk transport in these particular axons (see below and "Discussion"). Although some tubulovesicular profiles are present in intervening segments, by far, most cytomembranes, in the form of an anastomosing, tubular, smooth endoplasmic reticulum, are localized, in addition to mitochondria, in varicosities (Fig. 3). Most of these cytomembranes appear to be organized in a glomerulus-like arrangement. Some varicosities may contain membranes in the form of large vesicles as well (Fig. $3 b$ ), although large vesicles are more prevalent in small varicosities (Fig. 3, $a$ and c). In view of a preferential distribution of actin and spectrin to varicosities (see below), it is of interest that the cytomembranes are embedded in an amorphous matrix.

Motile behavior of varicosities. When viewed with time-lapse video, the varicosities exhibit dynamic axial movements that are 

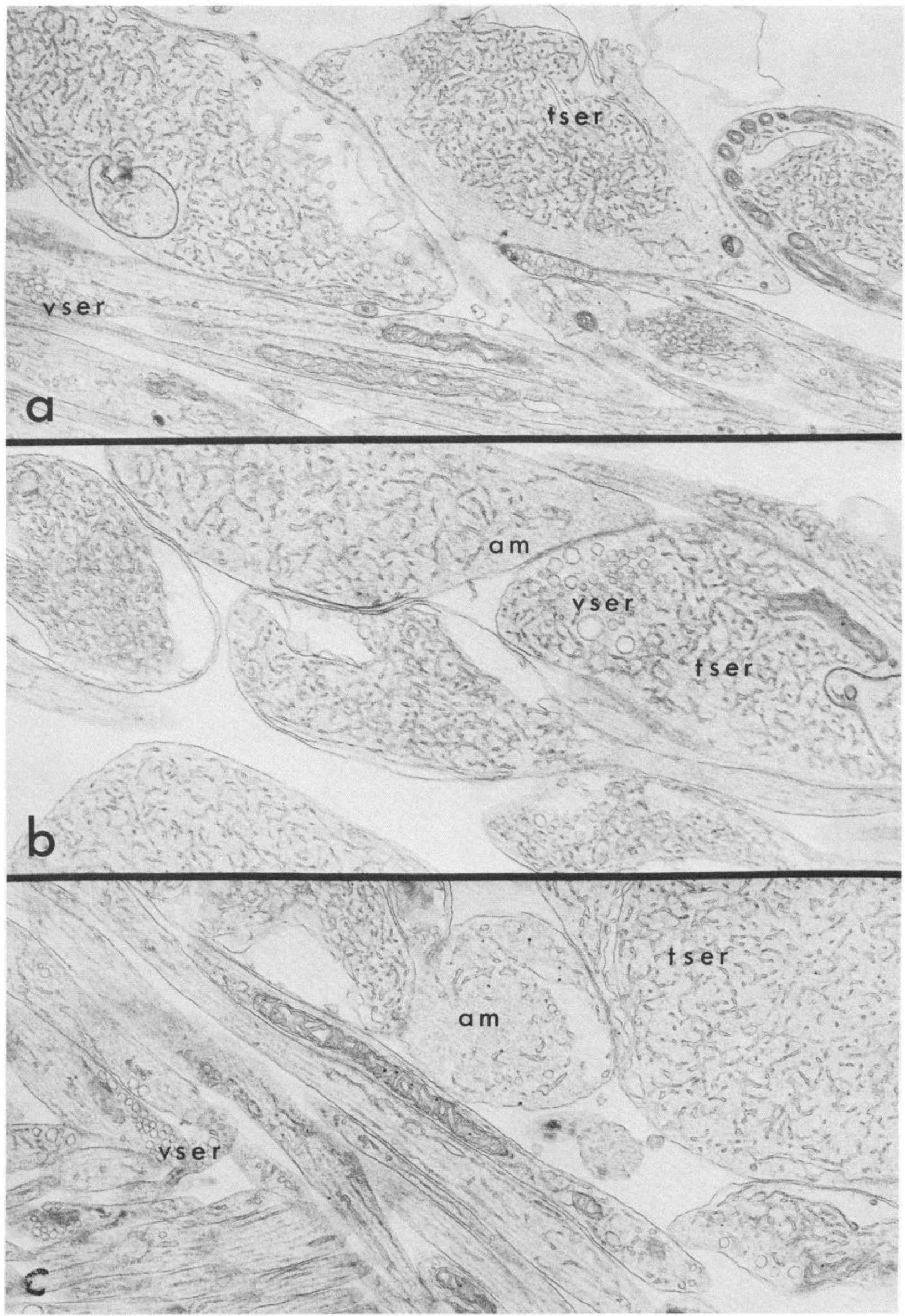

Figure 3. Electron micrographs of axonal varicosities. $a$ to $c$ are various examples of varicosities showing different packing densities and organization of tubular and vesicular elements of smooth endoplasmic reticululm. The amount of organization of cytomembranes suggest that it represents a mode of packaging for bulk transport. Note that tubulovesicular smooth endoplasmic reticulum appears embedded in an amorphous matrix. am, amorphous matrix; tser, tubular smooth endoplasmic reticulum; vser, vesicular smooth endoplasmic reticulum. Magnifications: $a, \times 16,400 ; b, \times 21,900 ; c, \times 19,300$. 


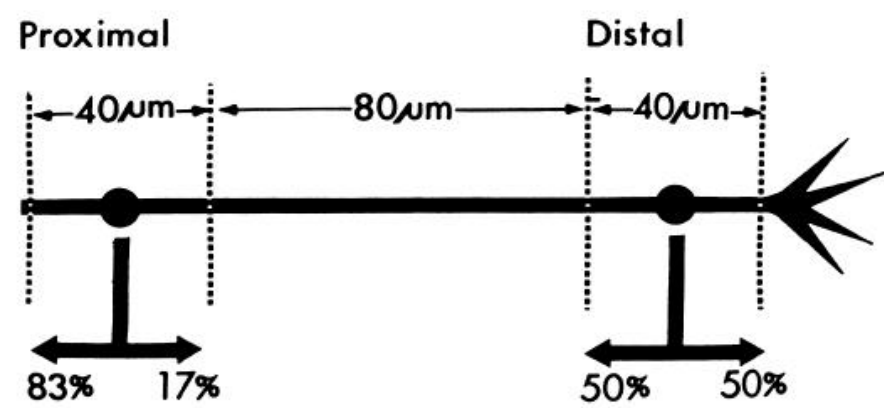

Figure 4. Frequency of bidirectional movements of varicosities in the distal elongating axon. The drawing depicts the relative frequencies of proximodistal and distoproximal movements of varicosities depending on their proximity to the growth cone in the distal axon.

saltatory in character. The saltations are quite variable in their excursions, averaging $33.8 \pm 22.8 \mu \mathrm{m}(n=13)$, and have a mean velocity during a saltation of $0.218 \pm 0.068 \mu \mathrm{m} / \mathrm{sec}$ at $22^{\circ} \mathrm{C}(n=$ 21). The onset of saltation is random in occurrence and does not affect neighboring stationary varicosities. The direction of movement depends upon the proximodistal location of the varicosity in the axon. Figure 4 depicts the frequency of proximodistal and distoproximal movements of varicosities, depending on their location in the distal axon; i.e., the distalmost $160 \mu \mathrm{m}$ of axons just proximal to growth cones. On the average, varicosities in the more proximal segment move in a retrograde direction more than $80 \%$ of the time whereas, in the distal segment, there is an equal probability that a varicosity moves in either a retrograde or an orthograde direction. The age of the explants examined ranged from 2 to 8 days and the data were pooled because there was no apparent age-related difference. Thus, although the determinants of directionality of movement are intrinsic and local, there is a rather sharp zone of transition close to the growth cone in which movement becomes biased in favor of a retrograde direction. It should be noted, however, that this conclusion is related to axons that are actively undergoing elongation, and it is not the case when axons undergo active retraction, at which time movement is all retrograde (see below).

At any given time, most varicosities appear stationary, but they usually exhibit a micro-oscillatory-like behavior. When a motile varicosity encounters a stationary one in its path it generally coalesces with it, forming a single, larger varicosity (Fig. 5). On infrequent occasions, we have observed a mobile varicosity bypassing a stationary one (Fig. 6), but this is unusual. After coalescence of mobile and stationary varicosities, the fused varicosity generally becomes stationary in the short run.

In addition to large varicosities, described above, there is a class of mobile inclusions we prefer to call intervening phase-dense (IPD) inclusions because they may not exhibit discrete varicose protrusions in the axon, but their moving dense profiles can be discerned in appropriate circumstances. We infer that they contain a similar composition of organelles as large varicosities because they frequently merge with varicosities, or they may be given off by a varicosity (see below). Other than their small size, they are distinguishable from varicosities by their mean saltation velocity, which is about an order of magnitude faster; i.e., $3.33 \pm 1.59 \mu \mathrm{m} / \mathrm{sec}$. This difference is reminiscent of the difference between axoplasmic transport groups II and I (see Grafstein and Forman, 1980).

IPD inclusions, although visible in proximal axons, are especially abundant in intervening segments between the growth cone and more proximal varicosities. Indeed, in distal segments, contiguous to growth cones of elongating axons, there may not be any obvious axial differentiation that would suggest discrete IPD inclusions (i.e., the appearance is that of a continuous column of axoplasm); however, the apparent uniformity can change rapidly to become nonuniform (see below). The particular example of a mobile IPD inclusion shown in Figure 7 is taken from an axon undergoing retraction (the growth cone is to the right, just out of the microscopic

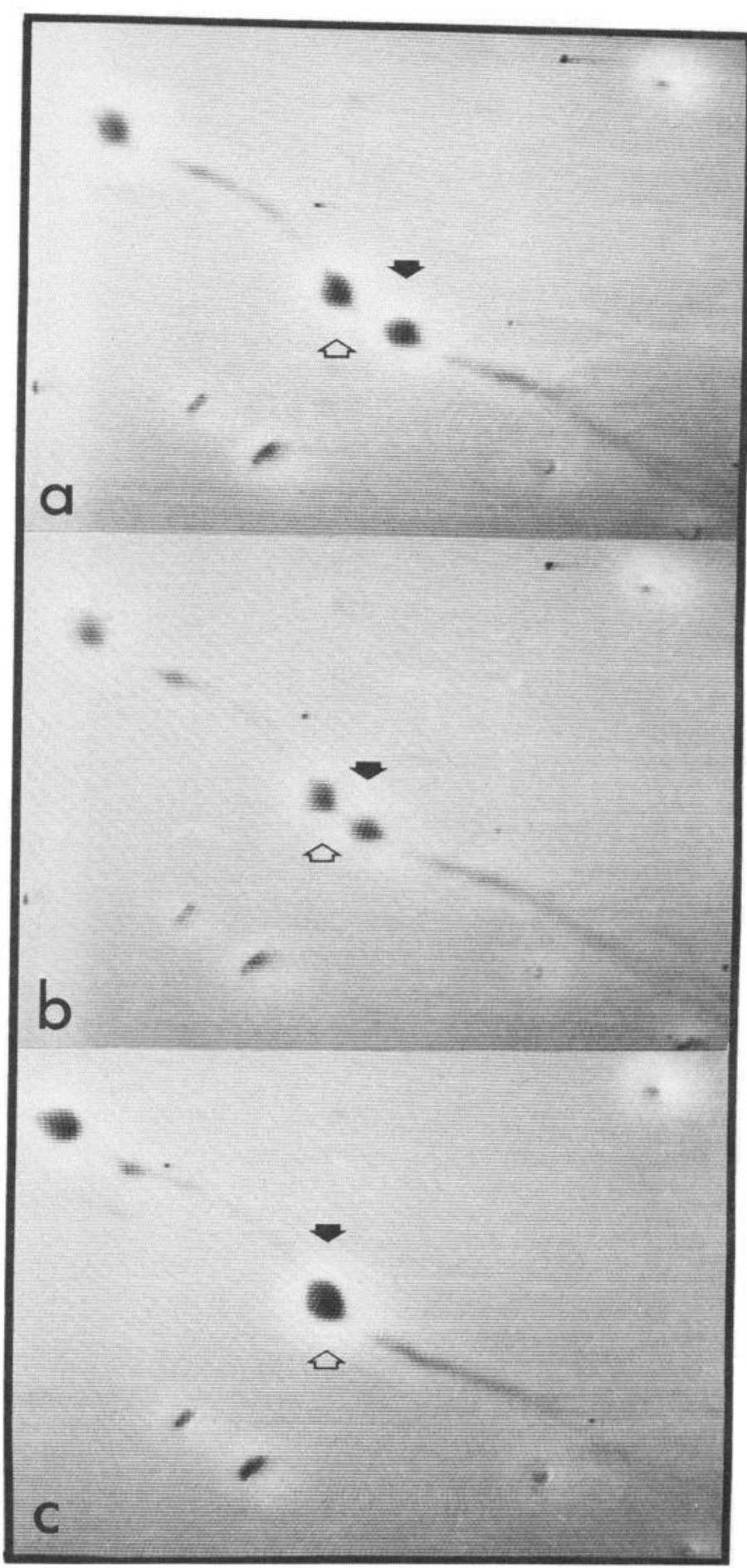

Figure 5. Coalescence of varicosities. This time-lapse video sequence shows the retrograde movement of a varicosity (solid arrows), which coalesced with a stationary varicosity (open arrows) when it collided with it; the product remained stationary for an undetermined period of time.

field); its transit proximally can be followed because of a visible varicose protrusion. In its rapid retrograde movement, it traverses multiple stationary varicosities, although its emergence from a given varicosity is frequently delayed; however, the delay in traversing a given varicosity does not appear to correlate necessarily with the size of the varicosity (i.e., it may be more rapid through a larger varicosity).

Other typical forms of behavior of IPD inclusions include merger with a stationary varicosity, and successive bidirectional movements 


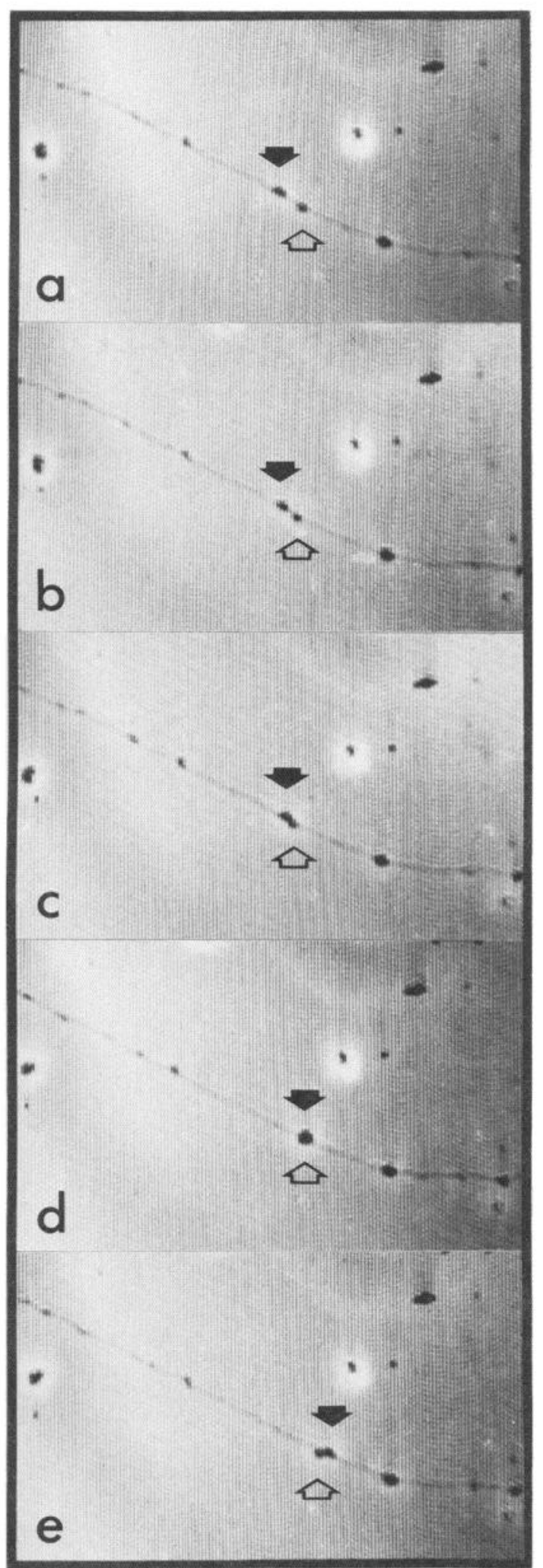

Figure 6. Bypass of varicosity. This time-lapse video sequence shows the orthograde movement of a varicosity (solid arrows), which bypasses a stationary varicosity (open arrows) after encountering it.

between stationary varicosities or between a stationary varicosity and a growth cone. Figure 8 shows a sequence in which there is a redistribution of axoplasmic material in a region contiguous to the growth cone of an elongating axon; some of the material is derived from a large varicosity, and the process is mediated by rapidly moving dense inclusions, which are not resolved in the figure but can be seen in the video monitor. The de novo formation of a varicosity, albeit transiently, by merging of IPD inclusions is also shown. Figure 9 shows the same axon after evacuation of the distal axon was triggered by adding $0.5 \mathrm{~mm}$ dibutyryl cyclic AMP and the phosphodiesterase inhibitor, $0.5 \mathrm{~mm}$ 3-isobutyl-1-methylxanthine, to the bathing medium. Evacuation is generally an antecedent to retraction. Material from the growth cone and contiguous distal axon is retrieved and incorporated into proximal varicosities, some of which form de novo as a result of the retrieval process. Varicosities and IPD inclusions, moving proximally, mediate the evacuation of the distal axon. Another example is shown in Figure 10, in which one of two branches undergoes retraction, while the other one from the same stem axon continues to elongate. The difference in thickness of the elongating and retracting branches is noteworthy, as well as the evacuation of axoplasmic material and the de novo formation of a large varicosity as a result of apparent "repackaging" of retrieved material. We conclude from these and other similar observations that rapidly moving IPD inclusions serve as vehicles for deploying membrane components to growth cones from varicosities during elongation, and redeploying membrane components from the distal axon to varicosities during axon retraction, as controlled by local extrinsic and intrinsic factors.

Dependence of intracellular motility on calcium. Fast axoplasmic transport in mature axons is known to be dependent on extracellular calcium (Ochs et al., 1977; Lavoie et al., 1979; Chan et al., 1980; Kanje et al., 1982). When explants were placed in a calcium-free medium, supplemented with $1 \mathrm{mM}$ EGTA, the growth cone rapidly involuted (i.e., filopidia retracted and the growth cone rounded up). In all other apparent respects, the axons appeared normal. Varicosities continued to undergo saltations in a calcium-free medium containing $1 \mathrm{~mm}$ EGTA for the period of observation (72 hr). However, movements of varicosities and phase-dense inclusions ceased rapidly (within $15 \mathrm{~min}$ ) when cadmium $(5 \mathrm{~mm}$ ) was substituted for calcium in the bathing medium. Barium $(10 \mathrm{~mm})$ and cobalt $(10 \mathrm{~mm})$ also blocked motility but required a longer period of incubation (15 to $25 \mathrm{~min}$ ); severing the axons in the presence of the calcium antagonist shortened the time of onset of an effect. Strontium did not block motility even when axons were severed in its presence in the bathing medium.

If the movement of varicosities and dense inclusions is dependent on intracellular calcium, as is indicated by the action of calcium antagonists, then the fact that varicosities continue to move while in a calcium-free medium for 3 days would seem to indicate that calcium must have been derived from intra-axonal stores. An attempt was made, therefore, to deplete the internal stores using the calcium ionophore A23187. The ionophore $\left(5 \times 10^{-6} \mathrm{M}\right)$ in a calcium-free medium containing $1 \mathrm{~mm}$ EGTA produced an arrest of movement of varicosities. These results provide evidence that calcium is essential for the bulk transport of material contained in the varicosities and IPD inclusions, and indicate further that internal stores serve as a requisite source of calcium to support intracellular motility.

Distribution of $\alpha$-spectrin, actin, and calmodulin in axons. We have shown by immunochemical and immunofluorescence techniques recently that an $\alpha$-spectrin-like protein is present in mature axons of the goldfish and rabbit (Koenig and Repasky, 1985). Indirect immunofluorescence of $\alpha$-spectrin in axonal fields indicates that it is also present in growing axons, where it is concentrated in varicosities, IPD inclusions, and growth cones (Figs. 11 and 14c). Within varicosities and growth cones, its distribution is frequently nonuniform. Thus, although it generally appears localized in the periphery of varicosities when focusing is in the plane of attachment to the substratum (Fig. 11a), consistent with its presumed linkage to the plasmalemma, it often appears also to be associated with internal structures of varicosities when the plane of focus is above the substratum, where the girth of varicosities is frequently maximal (Fig. $11, c$ and $d$ ). In growth cones, when regional differences in fluores- 

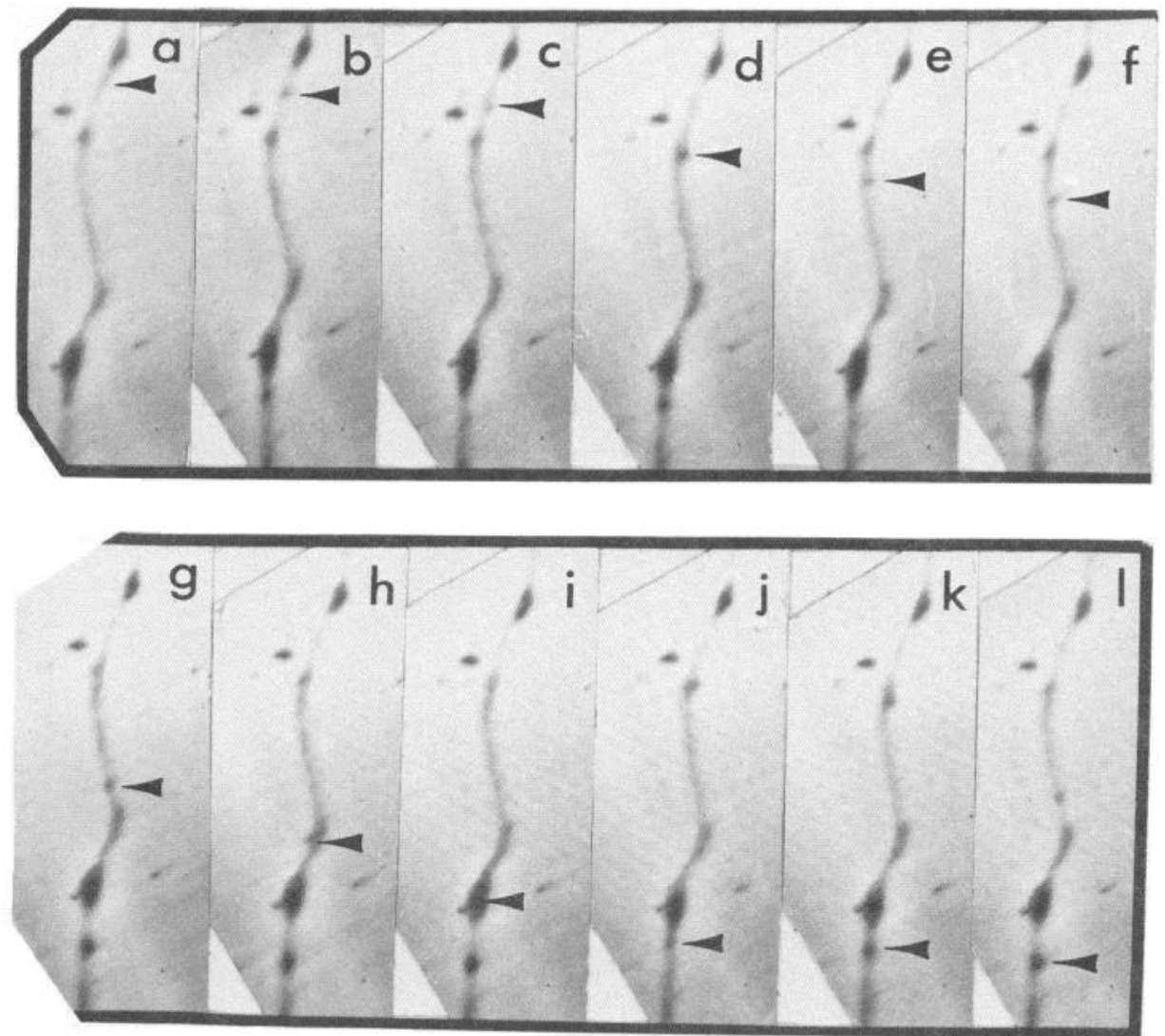

igure 7. Mobile IPD inclusion. This time-lapse to sequence shows a retrograde excursion of PD inclusion (arrowheads) over an interval of ut a minute. In its transit, the IPD inclusion erses other IPD inclusions and two varicosi-

cence intensity are evident, the more intense fluorescence is generally associated with the base and/or leading frontal portion of the growth cone (Fig. 11, e and f). Filopodia are visible as a result of $\alpha$ spectrin immunofluorescence, but they are not as sharply defined as they are when F-actin is labeled (see below).

Actin, labeled by indirect immunofluorescence, exhibits a distributional pattern that is very similar to that of $\alpha$-spectrin, in that varicosities, IPD inclusions, and growth cones exhibit the most intense fluorescence (Figs. 12 and 13). However, unlike spectrin, the distribution appears uniform throughout the varicosity even when the plane of focus is above the substratum (Fig. 12b). When rhodamine-conjugated phalloidin is also used to label F-actin selectively, the same structures show fluorescence, but there are large variations in form and intensity (Fig. 13). Thus, in varicosities, fluorescence due to phalloidin binding may appear granular and rather diffuse, with weak or moderate intensity (Figs. $13, b$ and $e$, and $14, b$, and $d$ ). There are instances when fluorescence due to phalloidin is discrete with strong intensity in preterminal axons; such fluorescent images may be punctate (Fig. 13e) or filar (Fig. 13f) structures that are randomly distributed. The latter are presumably bundles of actin filaments; however, the significance of the intensely fluorescent puncta is not clear, although they presumably represent foci of polymerized actin. As expected, most growth cones are strongly fluorescent when labeled with phalloidin (Figs. 13d and $14 b)$; in other structures, such as varicosities and IPD inclusions, there are wide variations in fluorescence intensity (cf. Figs. 13, $b, d$, and $e$, and $14, b, e$, and $f$ ). This may reflect a local dynamic modulation of the state of actin polymerization.

Similar to $\alpha$-spectrin and actin immunofluorescence, calmodulin immunofluorescence is strongest in varicosities, IPD inclusions, and growth cones (Fig. 14a). A comparison of fluorescence images due to immunolabeling of calmodulin and to phalloidin binding is shown in Figure 14, $a$ and $b$, respectively. A similar comparison between $\alpha$-spectrin and phalloidin is shown in Figure 14, $c$ and $d$, respectively. It is worth noting in the latter example that, although there is an apparent correspondence, there are also some subtle differences, particularly with respect to IPD inclusions.

\section{Discussion}

The movements of membraneous organelles have been studied in a number of isolated axonal systems using optical techniques in combination with time-lapse cinemicrography or video recording (for review, see Grafstein and Forman, 1980). In these axonal systems it is necessary to resolve individual axoplasmic organelles in order to monitor their movements. Regenerating axons of goldfish retinal ganglion cell in culture have motile varicosities that are large and readily visible on a microscopic scale. Varicosities are not unique to regenerating goldfish retinal ganglion cell axons in vitro but have also been reported in vivo as well (Murray, 1976).

The predominant structural inclusion of varicosities is a tubular smooth endoplasmic reticulum that is organized in an anastomosing glomerulus-like arrangement, although large vesicles may also be present to a variable extent in some instances. The cytomembranes appear to be embedded in an amorphous matrix, and given that varicosities are motile, it appears likely that these cytomembranes are in a "packaged" form for bulk bidirectional transport. This view is supported by direct observations indicating that they undergo bidirectional movement and are involved in reciprocal exchanges of material with the growth cone in elongating and in retracting axons (see below). Injection of rhodamine-conjugated wheatgerm agglutinin $(50 \mu \mathrm{g})$ into the vitreous of the eye (Margolis et al., 1981) 6 or $24 \mathrm{hr}$ before explanation results in labeling of inclusions of some varicosities when viewed after 3 days of outgrowth (unpublished observations). The latter findings indicate that cytomembranes in varicosities contain glycoconjugates and further support the view that they are destined for the distal axon where expansion of surface plasmalemma occurs (Bray, 1970, 1973; Pfenninger and MayliéPfenninger, 1981), or for the cell body.

The apparent bulk transport of large membranous aggregates in an axon is a novel circumstance, which may be related to the paucity 

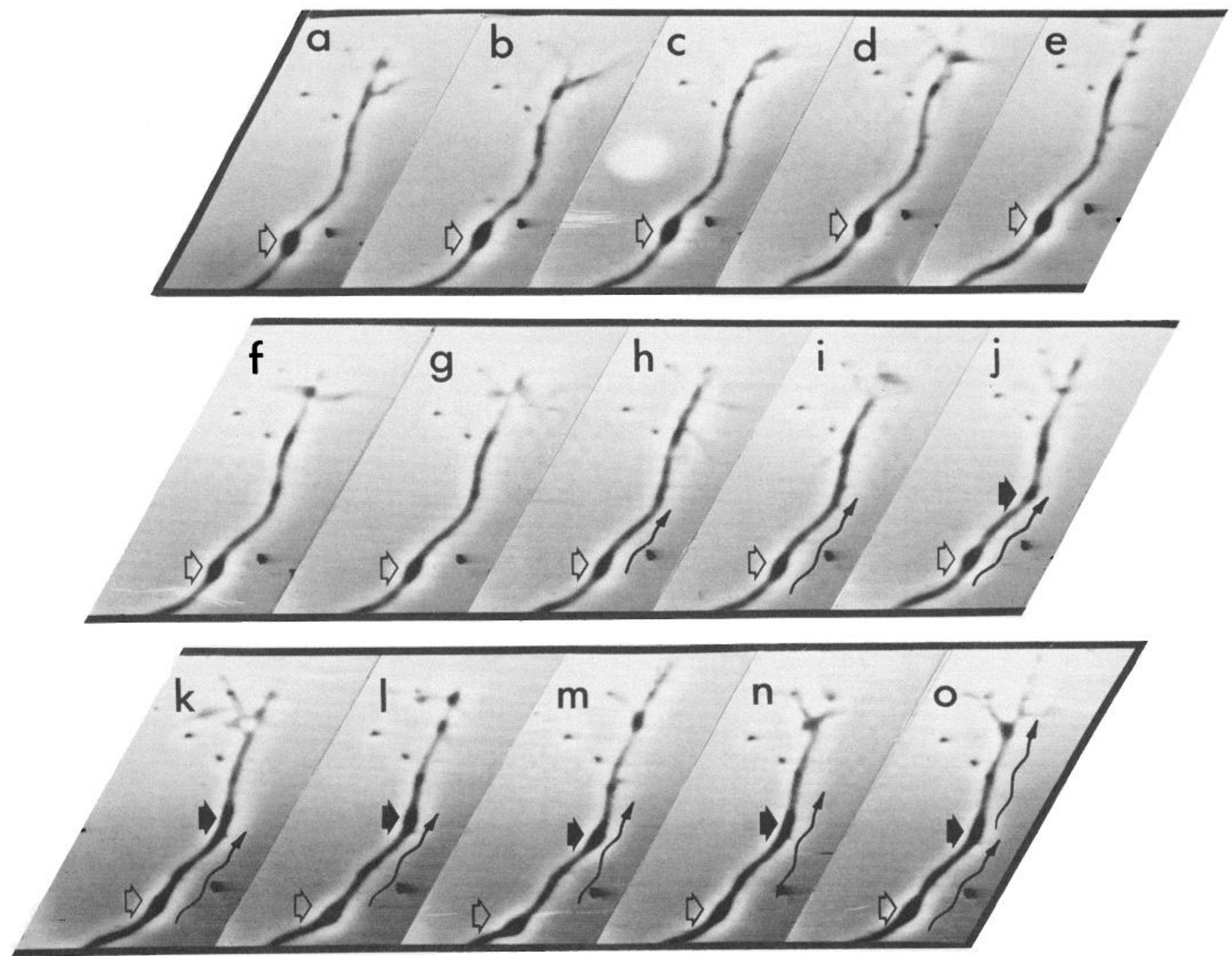

Figure 8. Proximodistal redistribution of axoplasmic material during elongation. This time-lapse video sequence shows a progressive proximodistal redistribution of axoplasmic material by IDP inclusions from a large varicosity (open arrows) toward the growth cone as the axon elongates. Identification and fate of individual IPD inclusions are difficult to discern because they merge rapidly, and photographic resolution is poor. The sequence also shows the de novo formation of a varicosity (solid arrows) by merger of IPD inclusions. Substantial proximodistal deployment is depicted by wavy arrows. The interval between frames is approximately $1 \mathrm{~min}$.

of neurofilaments in these immature axons as previously noted (Koenig and Adams, 1982). The relative lack of neurofilaments has also been reported in regenerating (RGC) axons in vivo (Murray, 1976). The apparent absence of a structured neurofilament domain may be equivalent to extending the microtubule domain throughout the axon's cross-section. According to Schnapp and Reese (1982), the microtubule domain delineates channels for the movement of organelles. Thus, it would appear that the absence of a threedimensional neurofilament cytoskeleton may favor the type of "bulk packaging" of cytomembranes that is characteristic of these axons. This may provide an explanation for the doubling in the fast transport rate and the 3-fold increase in amount transported, as reported for recently regenerated RGC axons in the goldfish (Grafstein and Murray, 1969).

Another class of visible motile structures in these growing axons is the IDP inclusion. Although IPD inclusions generally do not exhibit a varicose protrusion, their dense profiles can be discerned in the axon under favorable circumstances. We have not conducted a systematic electron microscopic study of IPD inclusions in the distal axon; therefore, we cannot comment on their contents. However, their faster rate of movement and their supply of material to the growth cone during elongation, or to proximal varicosities during retraction, are consistent with the assumption that they do contain cytomembranes. Thus, the net orthograde shuttling from proximal varicosities to growth cone during expansion of surface membrane and axon elongation and the net retrograde shuttling between growth cone and proximal varicosities during retrieval and axon retraction suggest that the IPD inclusions represent a mode by which material is rapidly deployed and exchanged in these axons.

The saltation velocity of the IPD inclusion is about an order of magnitude faster than that of the varicosity. Although extrapolation to mean transport rates based on radioactive labeling experiments in vivo is not justified, it seems appropriate, nevertheless, to point out the parallelism between the relative rates of the two mobile structural entities and those of transport groups I and II (see Grafstein and Forman, 1980). In any case, the movements of both varicosities and dense inclusions are calcium dependent. This conclusion is based in part on the actions of calcium antagonists such as cadmium, cobalt, and barium which block movement, and/or a calcium agonist such as strontium which does not arrest movement. These results are consistent with those reported in transport studies or radioactively labeled proteins in vertebrate nerves in vitro (Ochs et al., 1977; Lavoie et al., 1979; Chan et al., 1980; Kanje et al., 1982). Unlike mature axons, however, transport in RBC axons does not 

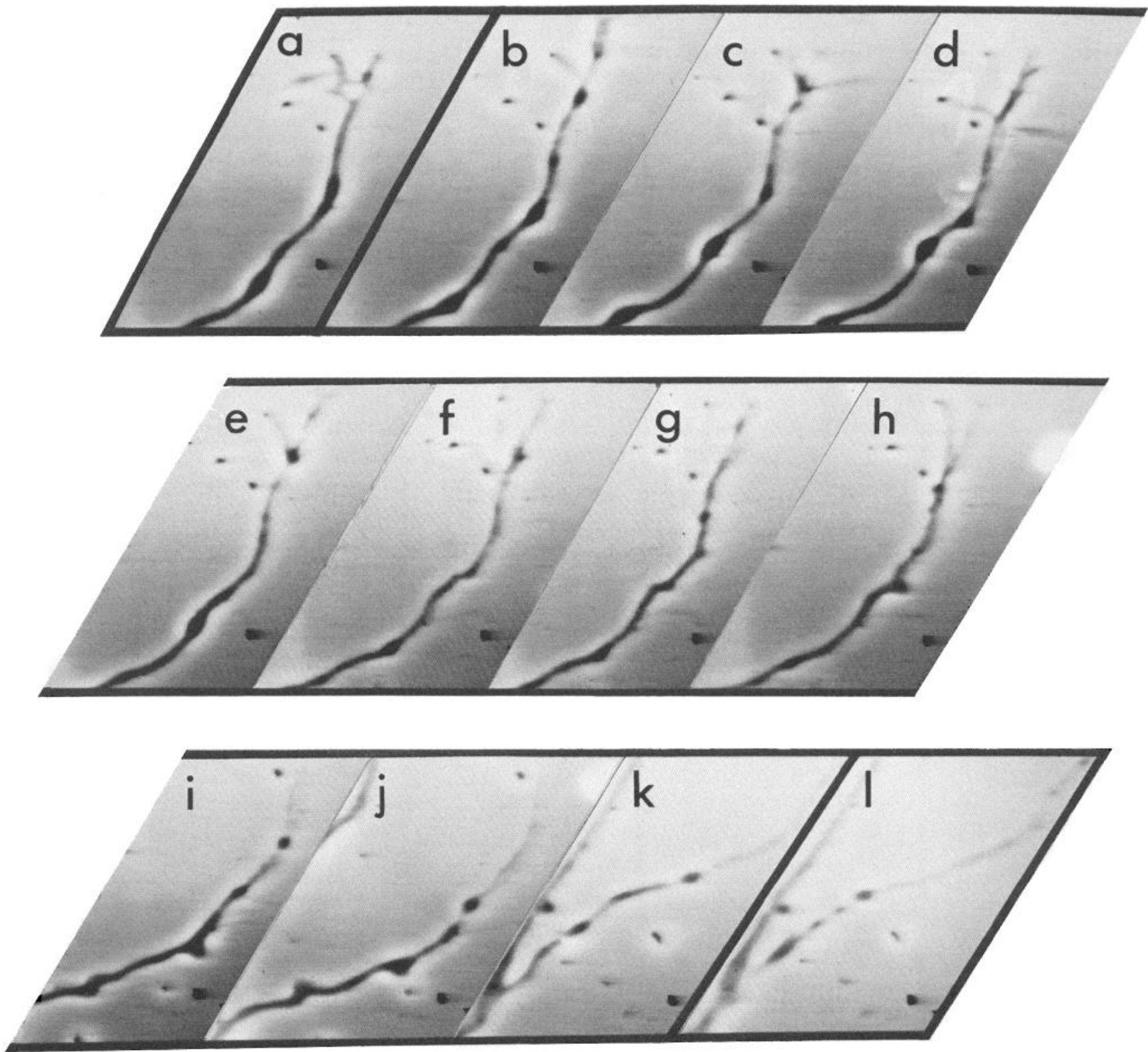

Figure 9. Distoproximal evacuation of axoplasmic material. This time-lapse video sequence shows retrieval and progressive distoproximal evacuation of axoplasmic material by IPD inclusions and varicosities. Evacuation is generally an antecedent to retraction. The interval between frames is approximately 2 $\min$.

depend on an extracellular source of calcium because movement is not halted for at least 3 days in a calcium-free medium containing EGTA. Such observations indicate that internal stores of calcium are sufficient to sustain intracellular transport. This inference was confirmed when movement was arrested after internal calcium was presumably depleted by bathing explants in a medium containing the calcium ionophore A23187 and EGTA.

The source of internal calcium required for transport is likely to be the cytomembranes that are being transported. There is ultrastructural evidence that the smooth endoplasmic reticulum in axons store calcium (Duce and Keen, 1978; Henkart et al., 1978), as well as evidence from several physiological studies that indicate the importance of a nonmitochondrial calcium-buffering organelle in preterminal and terminal axons for regulating internal concentrations of calcium (Brinley et al., 1977; Blaustein et al., 1978; Tiffert and Brinley, 1981). The fact that calcium appears to be essential for movement would suggest, furthermore, that it may serve to initiate a saltation. However, if movement is triggered by calcium, then it is likely that calcium is released locally and in the form of a transient which could then act as a second messenger and alter the state of the transport machinery locally. If calcium release were not transient, then motile varicosities or IPD inclusions would trigger the movement of neighboring stationary varicosities or IPD inclusions that they would encounter during their transit. The consequence of a collision between moving and stationary structures is generally a coalescence (varicosities and IPD inclusions; e.g., Fig. 6), or a by- or throughpass (mostly IPD inclusions; e.g., Figs. 7 and 8).

In the fluorescence cytochemistry experiments, our primary concern was to stabilize cytoskeletal organization during the preparative procedure without introducing artifact. We found that the presence of zinc in the fixation and Triton X-100 extraction steps to be helpful in achieving this goal, particularly since the aldehyde fixation was mild, so that immunoreactivity would be minimally affected (see "Materials and Methods"). A high zinc concentration in a physiological saline, however, slowly induces a change in morphology of varicosities after a delay of several minutes if an aldehyde fixative is not present. 

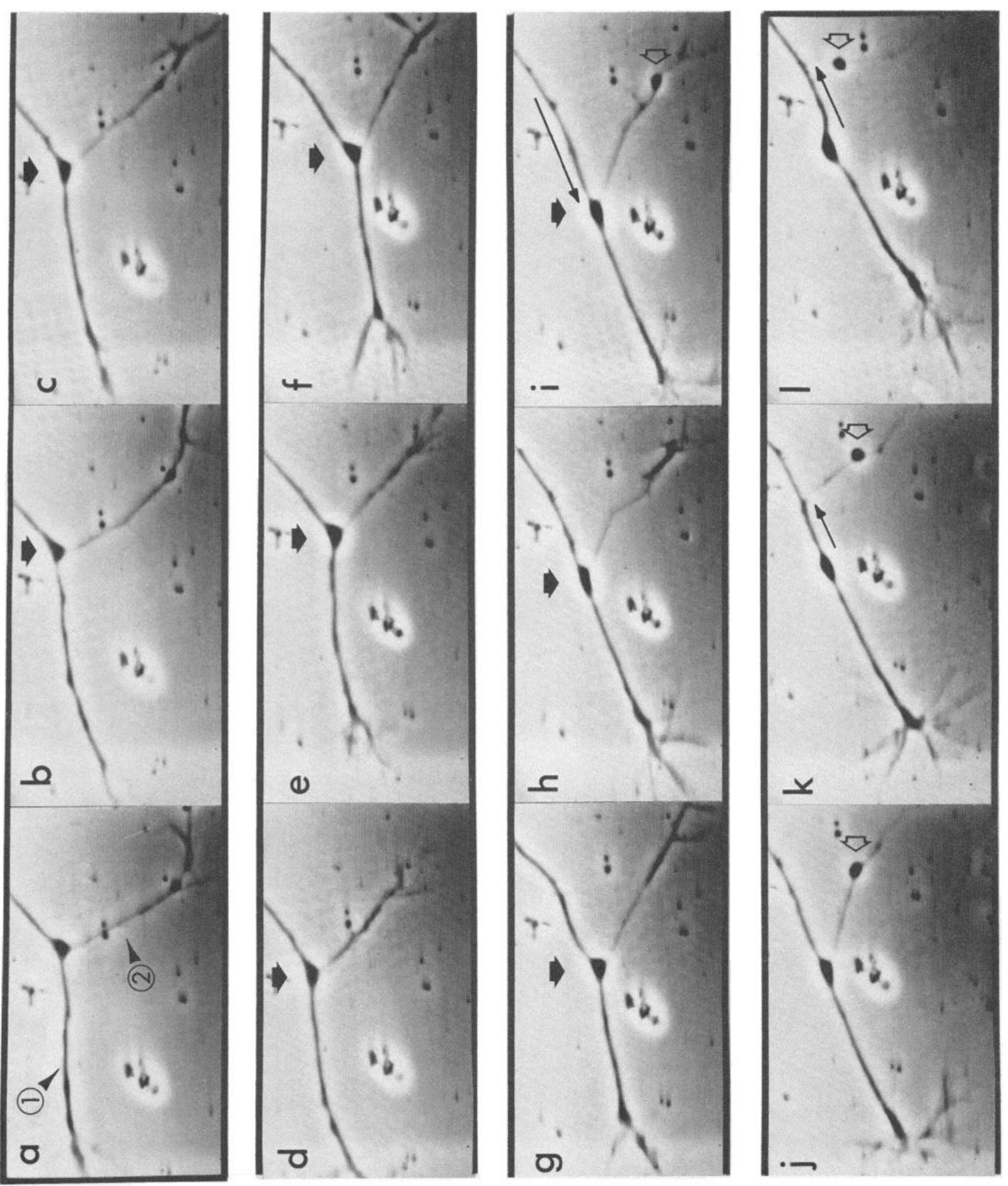

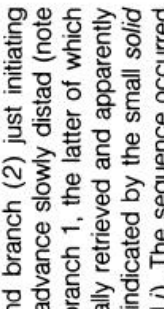

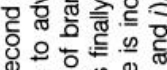

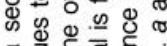
๙。

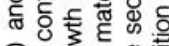

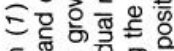

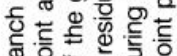

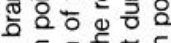

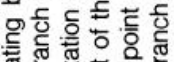

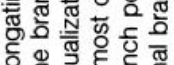

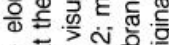

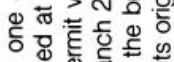

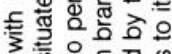

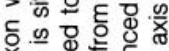
बड़्ष

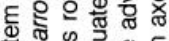
क人 in

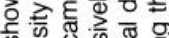
कs

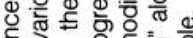

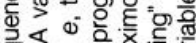

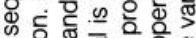

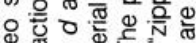

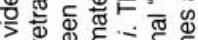

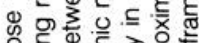

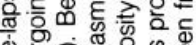

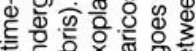

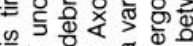

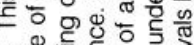

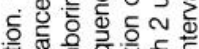

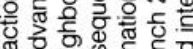
政 은

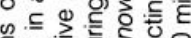

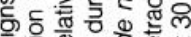

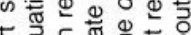

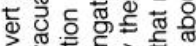
万人

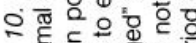
$0 \times 10$ 안응를 

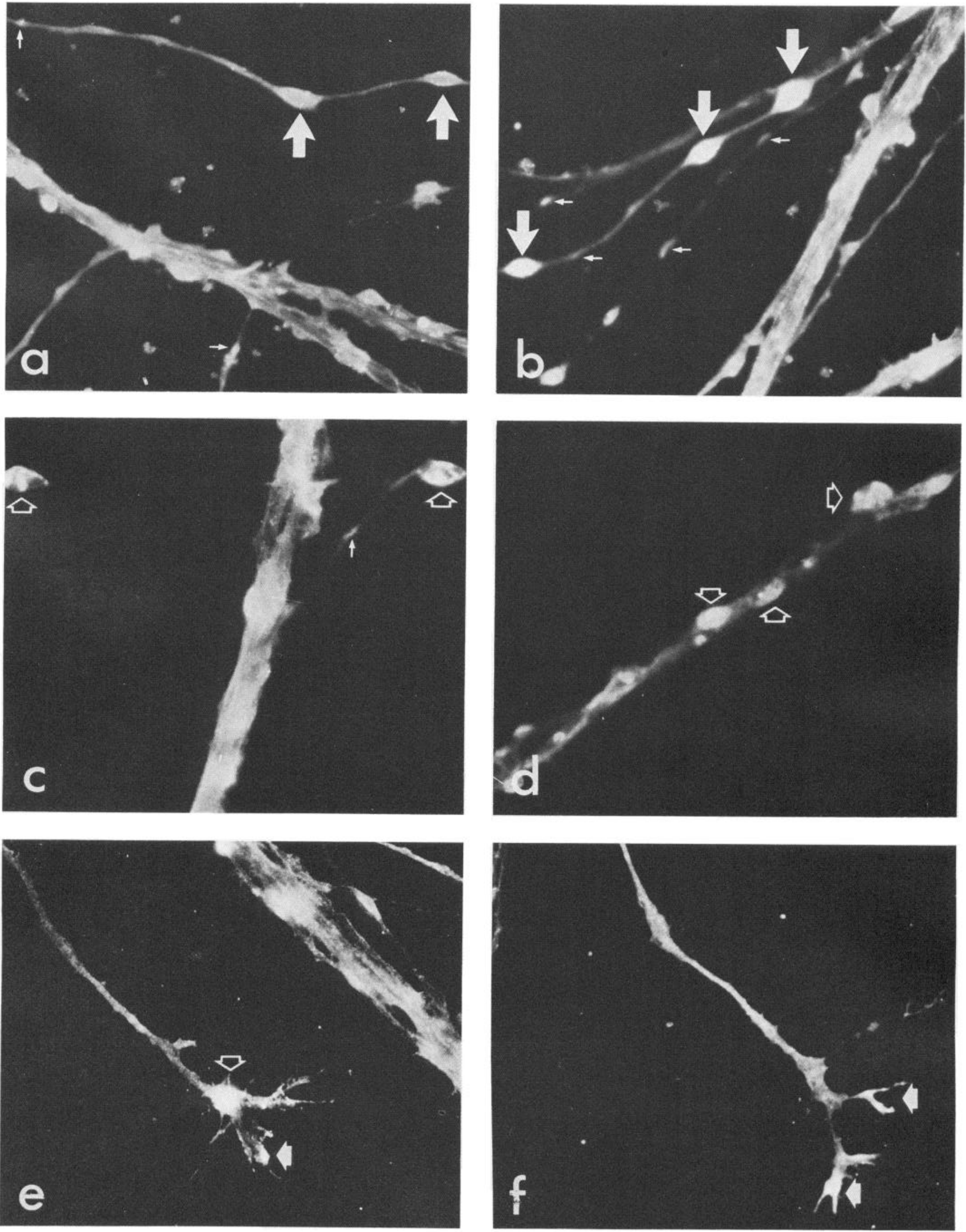

Figure 11. Localization of $\alpha$-spectrin in axonal structures. $\alpha$-Spectrin immunofluorescence is associated principally with varicosities (a to $d$; large arrows), IPD inclusions $(a, b$, and $c$; small arrows; see also Fig. 15, $e$ and $d)$, and growth cones ( $e$ and $f)$. When focused in the plane of the substratum ( $a$ and $b)$, the immunofluorescence appears diffuse and generally brighter around the perimeter; however, when focused above this plane, immunofluorescence generally appears nonuniform and associated with inclusions within the varicosity ( $c$ and $d$; open arrows). In growth cones, immunofluorescence is often associated with the base (e; open arrow) and with the advancing front ( $e$ and $f$; solid arrows). 

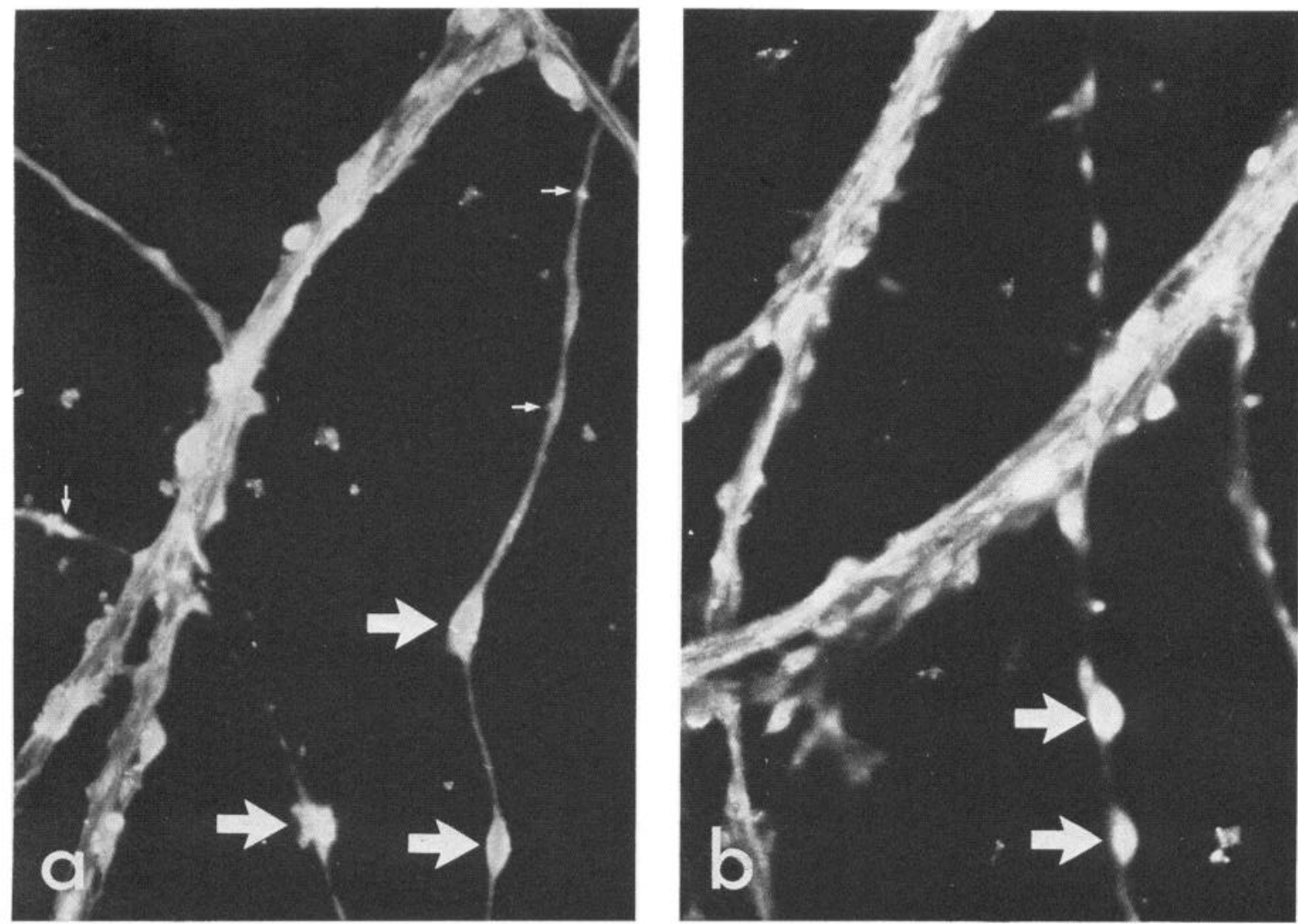

Figure 12. Localization of actin in preterminal axonal structures. Actin immunofluorescence is associated with varicosities ( $a$ and $b$; large arrows) and IPD inclusions (a; small arrows; see also Fig. 14, $a$ and $c)$. Unlike spectrin, immunofluorescence is diffuse and does not exhibit a nonuniform distribution in varicosities when the plane of focus is above the substratum (cf. b with Fig. 12, $c$ and $d$ ).

Actin, as shown by indirect immunofluorescence or by labeling with rhodamine-phalloidin, is distributed preferentially to varicosities, IPD inclusions, and growth cones. Qualitatively, there is a similarity in the distribution shown by immunofluorescence, which should reveal both $\mathrm{G}$ - and F-actin distributions, and that shown by phalloidin labeling, which should selectively label F-actin (Wieland, 1977). Although intense fluorescence due to phalloidin binding, in the forms of filar or punctate arrangements, are variable and sparsely distributed in the axons, weak to moderate intensity fluorescence is more widespread and rather characteristic of varicosities. Although it is difficult to draw any inferences about the significance of the variation and local differential intensity of phalloidin fluorescence as it may relate to the state of actin polymerization, preliminary studies clearly indicate that solation-gelation of the cytoskeleton of varicosities can be readily modulated by various experimental manipulations that usually involve changing $\left[\mathrm{Ca}^{2+}\right]_{\mathrm{i}}$ (unpublished observations), indicating that dynamic actin cytoskeletal structural transitions (for review, see Korn, 1982; Weeds, 1982) are probably ongoing and regulated locally.

The strategic localization of actin in varicosities and dense inclusions suggests that actin is likely to play a central role, perhaps in conjunction with spectrin (see below), in the "packaging" of cytomembranes in varicosities and dense inclusions, as well as in the mobility of these motile structures. Suggestive evidence that actin is probably involved in rapid axonal transport is provided by experiments in which microinjection of $\mathrm{F}$-actin depolymerizing substances into the giant neuron of Aplysia caused a blockade (Goldberg et al., 1980).

In mature axons that are isolated and stripped free of myelin sheath, $\alpha$-spectrin appears near the surface of the axon, where it is organized into a reticular network, except for morphologically differentiated nodes of Ranvier, where it appears as a dense band (Koenig and Repasky, 1985). In RGC axons, $\alpha$-spectrin, like actin, is distributed primarily to varicosities, IPD inclusions, and growth cones. In many growth cones, it appears more concentrated in the frontal leading portions of the growth cone, where possibly membrane may be inserted (Pfenninger and Maylié-Pfenninger, 1981). Although spectrin appears to be localized near the surface of many varicosities, it also seems to be associated with inclusions of the varicosity. If this is the case, then spectrin, along with actin, could play a role in the "packaging" and/or transport of the tubular arrangement of cytomembranes, since spectrin presumably would be cross-linked to putative precursor plasma membrane, and has been shown to be an F-actin-binding protein (Brenner and Korn, 1979; Levine and Willard, 1981; Burridge et al., 1982; Glenny et al., 1982).

Axoplasmic transport of a brain spectrin heterodimer, that was later called fodrin (Levine and Willard, 1981), was first described in Willard's laboratory (Lorenz and Willard, 1978; Levine and Willard, 1980), at which time it was observed to be associated with all but the most rapid of the transport groups (i.e., groups II, III, IV, and V). Indeed, it was the presence of fodrin in multiple transport groups that led Levine and Willard (1980) to propose that, by virtue of its location at the inner surface of the plasma membrane, it might serve to link proteins and organelles to a cortical force-generating system in the axon and thereby play a role in the transport process. This view is not inconsistent with our observations in the present study. However, our observations of regenerating axons in vitro lead us to focus more sharply on a possible structural relationship between a spectrin-actin system and the cytomembranes (i.e., putative plasma membranes) that appear in a packaged form and undergo transport. It is clear that it will be necessary to seek confirmation at the electron microscopic level of our inference that spectrin may be associated with cytomembranes of varicosities and IPD inclusions.

The distribution of calmodulin immunofluorescence parallels that 

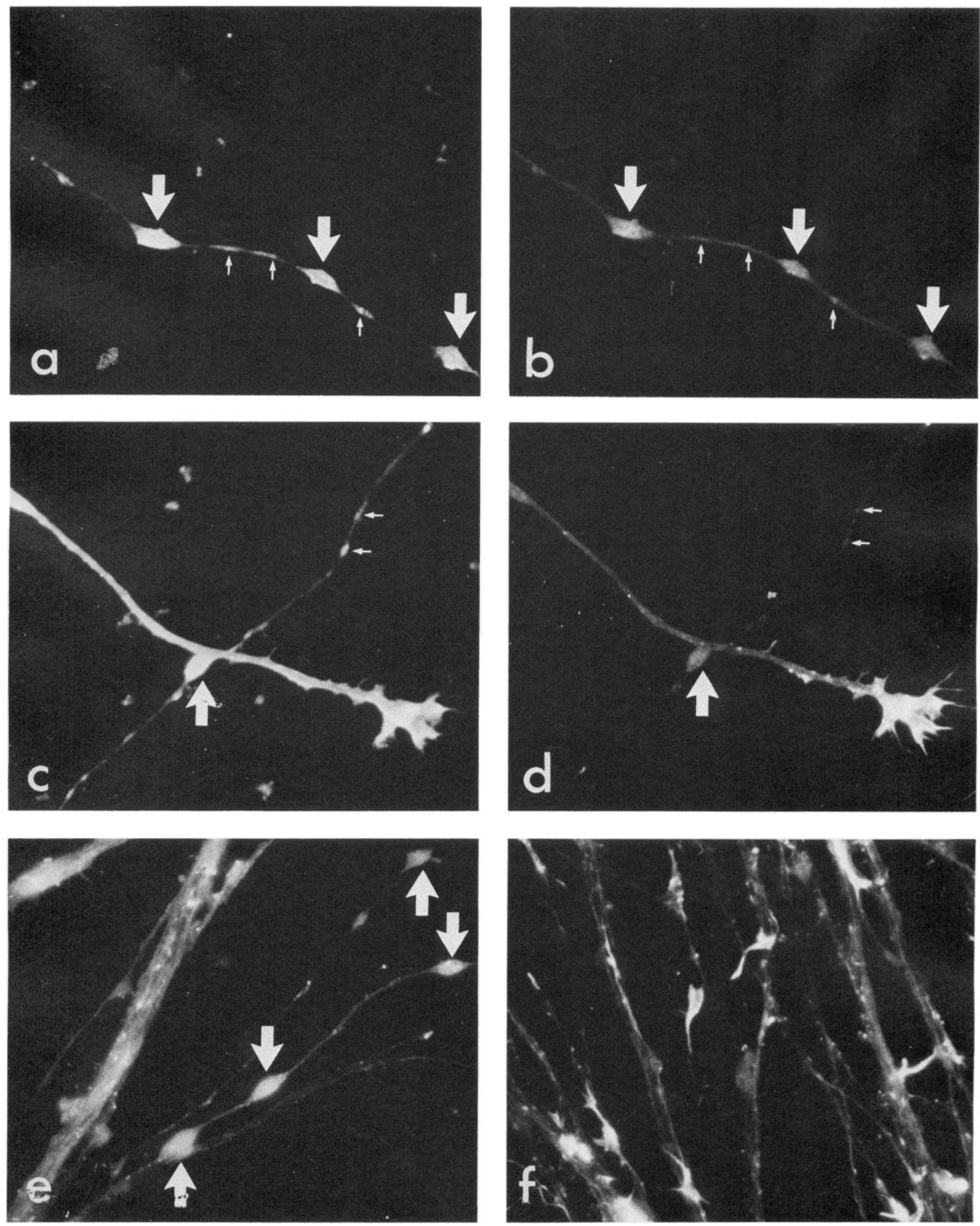

Figure 13. Double labeling of actin with antiserum and phalloidin. A comparison of fluorescein immunofluorescence (a and $c)$ with rhodamine-conjugated phalloidin fluorescence $(b, d$, and $e$; see also Fig. $15, b$ and $d$ ) shows wide differences among varicosities (large arrows) and IPD inclusions (small arrows) with respect to the relative amount of F-actin. Fluorescence due to phalloidin binding $(d)$ is generally strong in growth cones (d; see also Fig. $15 b)$, and filopodia are better defined than those stained by immunofluorescence (cf. $c$ and $d$ ). Strong fluorescence due to phalloidin binding may appear randomly in preterminal axons in either a punctate $(e)$ or filar $(f)$ form. 

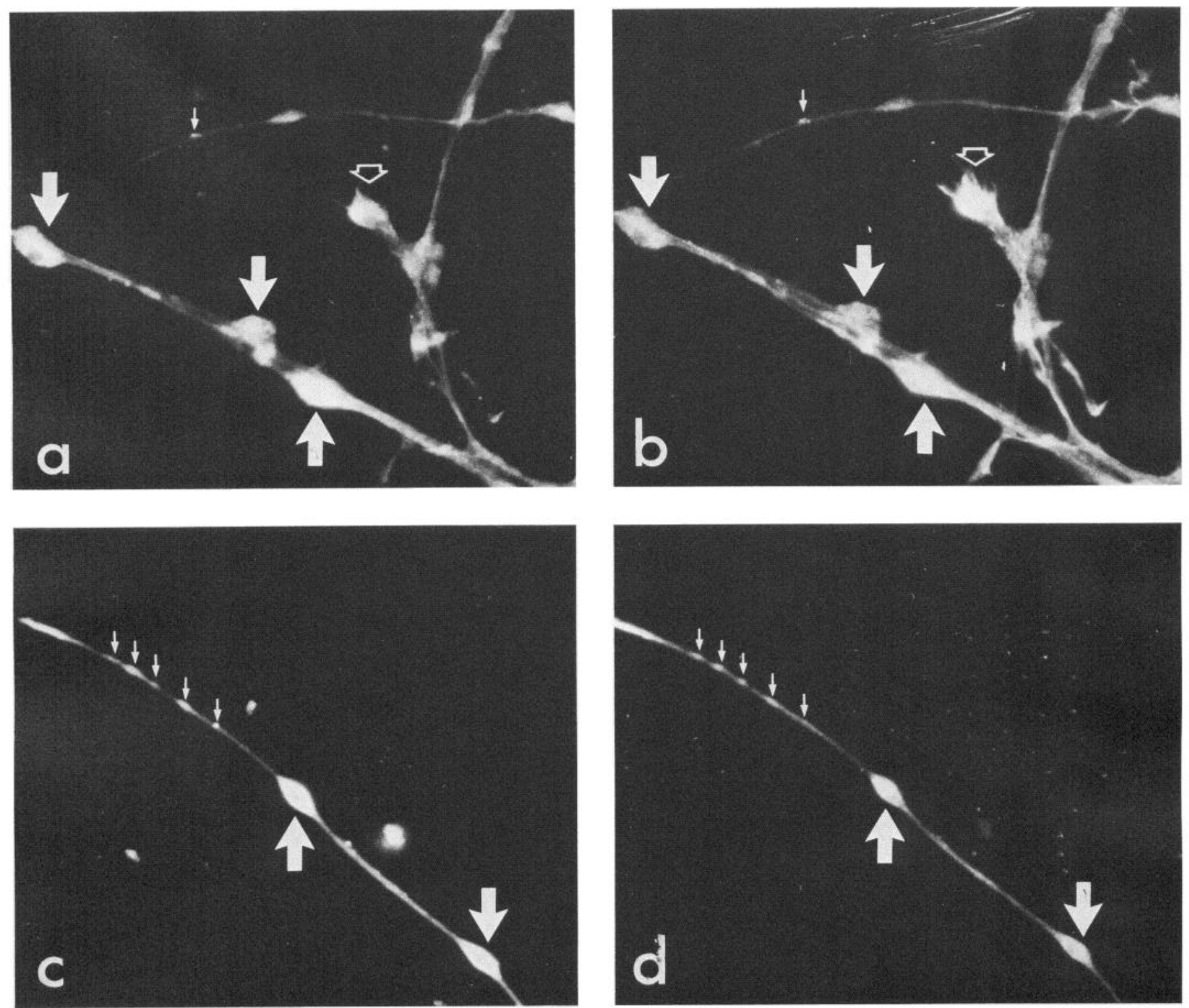

Figure 14. Double labeling of calmodulin or $\alpha$-spectrin with phalloidin. A comparison is made of fluorescein immunofluorescence of calmodulin (a) or of $\alpha$-spectrin (c) with rhodamine-conjugated phalloidin fluorescence ( $b$ and $d$ ). Calmodulin ( $a$ ), like spectrin and actin, is localized largely to varicosities (large arrows), IPD inclusions (small arrows), and growth cones (open arrows). IPD inclusions are well defined in the retracting axon shown in $c$ and $d$; subtle differences in the $\alpha$-spectrin immunofluorescence and phalloidin fluorescence images can be seen and may reflect differences in the polymerized state of actin.

of actin and of spectrin in RGC axons. Whereas the $\alpha$-spectrin subunit has been shown to be a calmodulin-binding protein (Kakiuchi et al., 1982; Glenney et al., 1982a, b; Palfrey et al., 1982), in vitro studies indicate that binding of F-actin is not calmodulin dependent (Glenney et al., 1982). In contrast, a recent study has provided evidence that in the presence of a soluble factor from brain, binding of F-actin by spectrin is blocked by calcium-calmodulin (Sobue et al., 1983). What significance, if any, this type of modulation could have on the "packaging" and/or "transport" of cytomembranes remains to be determined.

As a final point, the present study raises an issue that appears somewhat paradoxical. Our observations of motile varicosities and IPD inclusions indicate that actin, spectrin, and calmodulin are cotransported with cytomembranes when these structures undergo saltation. On the basis of radioactive labeling experiments, however, actin, spectrin, and calmodulin are not transported with membranes, but with cytoskeletal and soluble components that are associated with slow transport (slow components b or transport group IV; Lorenz and Willard, 1978; Black and Lasek, 1979; Willard et al., 1979; Erickson et al., 1980; Brady et al., 1981) (although, as noted above, spectrin (fodrin) is an exception insofar as it is associated with transport group II). However, since actin is known to undergo treadmilling, at least in vitro (see Korn, 1982), the paradox could be explained if one assumes that the association of actin (or calmodulin) subunits with the transport machinery is transitory and that the subunits, in the course of time, would exchange with the stationary, soluble actin (or calmodulin) pool. If this were the case, then the apparent slow transport of radioactively labeled actin (or calmodulin) would reflect successive cycles of treadmilling and/or exchange with a stationary pool, and a radioactive peak would appear to be gradually displaced with time.

Note added in proof. Subsequent calcium depletion experiments with A23187 and EGTA have shown that, whereas movements of all varicosities throughout the axonal field are arrested, movements of IPD inclusions only in distal axons are arrested. The significance of sustained movements of IPD inclusions under calcium-depleting conditions in proximal axons is at present unknown. 


\section{References}

Allen, R. D., R. J. Lasek, S. P. Gilbert, A. J. Hodge, and C. K. Govind (1982a) Fast axonal transport in lobster axons. Biol. Bull. 163: 379-380.

Allen, R. D., J. Metuzals, I. Tasaki, S. T. Brady, and S. P. Gilbert (1982b) Fast axonal transport in squid giant axon. Science 218: 1127-1129.

Black, M. M., and R. J. Lasek (1979) Axonal transport of actin: Slow component $b$ is the principal source of actin for the axon. Brain Res. 171: 401-413.

Blaustein, M. P., R. W. Ratzlaff, N. C. Kendrick, and E. S. Schweitzer (1978) Calcium buffering in presynaptic nerve terminals. I. Evidence for involvement of a nonmitochondrial ATP-dependent sequestration mechanism. J. Gen. Physiol. 72: 15-41.

Brady, S. T., M. Tytell, K. Heriot, and R. J. Lasek (1981) Axonal transport of calmodulin-A physiologic approach to identification of long-term associations between proteins. J. Cell Biol. 89: 607-614.

Bray, D. (1970) Surface movements during the growth of single explanted neurons. Proc. Natl. Acad. Sci. U. S. A. 65: 905-910.

Bray, D. (1973) Model for membrane movements in the neural growth cone. Nature 244: 93-96.

Brenner, S. L., and E. D. Korn (1979) Spectrin-actin interaction. J. Biol. Chem. 254: 8620-8627.

Breuer, A. C., C. M. Christian, M. Henkart, and P. G. Nelson (1975) Computer analysis of organelle translocation in primary neuronal cultures and continuous cell lines. J. Cell Biol. 65: 562-576.

Brinley, F. J., Jr., T. Tiffert, A. Scarpa, and L. J. Mullins (1977) Intracellular calcium buffering capacity in isolated squid axons. J. Gen. Physiol. 70. 355-385

Burridge, K., T. Kelly, and P. Mangeat (1982) Nonerythrocyte spectrins: Actinmembrane attachment proteins occurring in many cell types. J. Cell Biol. 95: $478-486$.

Chafouleas, J. G., J. R. Dedman, R. P. Munjaal, and A. Means (1979) Calmodulin: Development and application of a sensitive radioimmunoassay. J. Biol. Chem. 252: 10262-10267.

Chan, S. -Y., S. Ochs, and R. M. Worth (1980) The requirement for calcium ions and the effect of other ions on axoplasmic transport in mammalian nerve. J. Physiol. (Lond.) 301: 477-504.

Cooper, P. D., and R. S. Smith (1974) The movement of optically detectable organelles in myelinated axons of Xenopus laevis. J. Physiol. (Lond.) 242 . 77-97.

Duce, I. R., and P. Keen (1978) Can neuronal smooth endoplasmic reticulum function as a calcium reservoir? Neuroscience $3: 837-848$.

Erickson, P. F., K. B. Seamon, B. W. Moore, R. S. Lasher, and L. N. Minier (1980) Axonal transport of the $\mathrm{Ca}^{++}$-dependent protein modulator of $3^{\prime}: 5^{\prime}$ cyclic-AMP phosphodiesterase in the rabbit visual system. J. Neurochem. 35: $242-248$.

Forman, D. S., and W. G. Shain, Jr. (1981) Batrachotoxin blocks saltatory organelle movement in electrically excitable neuroblastoma cells. Brain Res. 211: 242-247

Forman, D. S., A. L. Padjen, and G. R. Siggins (1977a) Axonal transport of organelles visualized by light microscopy: Cinemicrographic and computer analysis. Brain Res. 136: 215-226.

Forman, D. S., A. L. Padjen, and G. R. Siggins (1977b) Effect of temperature on the rapid retrograde transport of microscopically visible intra-axonal organelles. Brain Res. 136: 215-226.

Forman, D. S., K. J. Brown, and D. R. Livengood (1983) Fast axonal transport in permeabilized lobster giant axons is inhibited by vanadate. J. Neurosci. 3: $1279-1288$.

Glenney, J. R., Jr., P. Glenney, and K. Weber (1982) F-actin-binding and cross-linking properties of porcine brain fodrin, a spectrin-related molecule. J. Biol. Chem. 257: 9781-9797.

Goldberg, D. J., D. A. Harris, B. W. Lubit, and J. H. Schwartz (1980) Analysis of the mechanism of fast axonal transport by intracellular injection of potentially inhibitory macromolecules: evidence for a possible role of actin filaments. Proc. Natl. Acad. Sci. U. S. A. 77: 7448-7452.

Grafstein, B., and D. S. Forman (1980) Intracellular transport in neurons. Physiol. Rev. 60: 1167-1283.

Grafstein, B., and M. Murray (1969) Transport of protein in goldfish optic nerve during regeneration. Exp. Neurol. 25: 494-508.

Heacock, A. M., and B. W. Agranoff (1977) Clockwide growth of neurites from retinal explants. Science 198: 64-66.

Henkart, M. P., T. S. Reese, and F. J. Brinley, Jr. (1978) Endoplasmic reticulum sequesters calcium in the squid giant axon. Science 202: 13001303.

Hooper, J. E., and R. B. Kelly (1984) Calmodulin is tightly associated with synaptic vesicles independent of calcium. J. Biol. Chem. 259: 148-153.

Johnson, J. E., and J. E. Turner (1982) Growth from regenerating goldfish retinal cultures in absence of serum or hormonal supplements: Tissue extract effects. J. Neurosci. Res. 8: 315-329.

Kakiuchi, S., K. Sobue, K. Kanda, K. Morimoto, S. Tsukita, H. Ishikawa, and M. Kurokawa (1982) Correlative biochemical and morphological studies of brain calspectrin: A spectrin-like calmodulin-binding protein. Biomed. Res. 3: $400-410$.

Kanje, J., A. Edström, and P. Ekström (1982) Divalent cations and fast axonal transport in chemically desheathed (Triton X-treated) frog sciatic nerve. Brain Res. 241: 67-74.

Kirkpatrick, J. B., J. J. Bray, and S. M. Palmer (1972) Visualization of axoplasmic flow in vitro by Nomarski microscopy. Comparison to rapid flow of radioactive proteins. Brain Res. 43: 1-10.

Koenig, E., and P. Adams (1982) Local protein synthesizing activity in axonal fields regenerating in vitro. J. Neurochem. 39: 386-400.

Koenig, E., and E. A. Repasky (1985) A regional analysis of $\alpha$-spectrin in the isolated Mauthner neuron and in isolated axons of the goldfish and rabbit. J. Neurosci. 5: 705-714.

Koles, Z. J., K. D. McLeod, and R. S. Smith (1982) A study of the motion of organelles which undergo retrograde and anterograde rapid axonal transport in Xenopus. J. Physiol. (Lond.) 323: 469-484.

Korn, E. D. (1982) Actin polymerization and its regulation by proteins from nonmuscle cells. Physiol. Rev. 62: 672-737.

Landreth, G. E., and B. W. Agranoff (1976) Explant culture of adult goldfish retina: Effect of prior optic nerve crush. Brain Res. 118: 299-303.

Lavoie, P. -A., F. Bolen, and R. Hammerschlag (1979) Divalent cation specificity of the calcium requirement for fast transport of proteins in axons of desheathed nerves. J. Neurochem. 32: 1745-1751.

Levine, J., and M. Willard (1980) The composition and organization of axonally transported proteins in the retinal ganglion cells of the guinea pig. Brain Res. 194: 137-154.

Levine, J., and M. Willard (1981) Fodrin: Axonally transported polypeptides associated with the internal periphery of many cells. J. Cell Biol. 90: 63t643.

Lorenz, T., and M. Willard (1978) Subcellular fractionation of intra-axonally transported polypeptides in the rabbit visual system. Proc. Natl. Acad. Sci. U. S. A. 75: 505-509.

Margolis, T. P., C. M. -F. Marchand, H. B. Kistler, Jr., and J. H. LaVail (1981) Uptake and anterograde axonal transport of wheat germ agglutinin from retina to optic tectum in the chick. J. Cell Biol. 89: 152-156.

Murray, M. (1976) Regeneration of retinal axons into goldfish optic tectum. J. Comp. Neurol. 168: 175-196.

Ochs, S., R. M. Worth, and S. -Y. Chan (1977) Calcium requirement for axoplasmic transport in mammalian nerve. Nature 270: 748-750.

Palfrey, H. C., W. Schiebler, and P. Greengard (1982) A major calmodulinbinding protein common to various vertebrate tissues. Proc. Natl. Acad. Sci. U. S. A. 79: $3780-3784$.

Pfenninger, K. H., and M. F. Maylié-Pfenninger (1981) Lectin labeling of sprouting neurons. II. Relative movement and appearance of glycoconjugates during plasmalemmal expansion. J. Cell Biol. 89: 547-559.

Repasky, E. A., B. L. Granger, and E. Lazarides (1982) Widespread occurrence of avian spectrin in nonerythroid cells. Cell 29: 821-833.

Rodriguez, J., and F. Deinhardt (1960) Preparation of a semipermeant mounting medium for fluorescent antibody studies. Virology 12: 316-317.

Schnapp, B. J., and T. S. Reese (1982) Cytoplasmic structure in rapid-frozen axons. J. Cell Biol. 94: 667-679.

Smith, B. H. (1971) Neuroplasmic transport in the nervous system of the cockroach Periplaneta americana. J. Neurobiol. 2: 107-118.

Sobue, K., K. Kanda, J. Adachi, and S. Kakiuchi (1983) Calmodulin-binding proteins that interact with actin filaments in a $\mathrm{Ca}^{++}$-dependent flip-flop manner: Survey in brain and secretory tissues. Proc. Natl. Acad. Sci. U. S. A. $80: 6868-6871$

Tiffert, T., and F. J. Brinley, Jr. (1981) In situ accumulation of calcium by organelles of squid axoplasm. Cell Calcium 2: 89-104.

Weeds, A. (1982) Actin-binding proteins-Regulators of cell architecture and motility. Nature 296: 811-816.

Wieland, T. (1977) Modification of actins by phallotoxins. Naturwissenschaften 64: 303-309.

Willard, J., M. Wiseman, J. Levine, and P. Skene (1979) Axonal transport of actin in rabbit retinal ganglion cells. J. Cell Biol. 81: 581-591. 